\title{
Comparación de herramientas quimiométricas de clasificación para la identificación de grasa perirrenal en corderos
}

\author{
Agudo, B. '; Delgado, J.V.2; López, M.M. ${ }^{3}$ y Rodríguez, P.L.4@
}

'Ovino del Suroeste Soc. Coop. Ltda. (OVISO), Villanueva de la Serena, Badajoz, España.

2Departamento de Producción Animal, Facultad de Veterinaria, Universidad de Córdoba, España.

3Departamento de Producción Animal, CYCITEX, Finca La orden, Gobierno de Extremadura, España.

${ }^{4}$ Departamento de Producción Animal y Ciencia de los Alimentos, Facultad de Veterinaria de Cáceres, España.

\section{PALABRAS CLAVE ADICIONALES}

Cordero.

Grasa perirrenal.

NIRS.

Clasificación no supervisada/supervisada.

\section{RESUMEN}

En el presente trabajo se prueba la capacidad de la técnica NIRS para clasificar correctamente dos tipos de grasa perirrenal de corderos provenientes de distintos tipos de alimentación. Se combinan distintos tratamientos espectrales (Asorbancia-A, Reflectancia-R, A-EMSC, R-EMS) con distintas técnicas de clasificación no supervisada (PCA-Projection) y supervisada SIMCA, PLS-DA, LDA, C-SVM. Los resultados indican que los espectros sin tratar, contienen información valiosa en términos de clasificación, a pesar de la dispersión luminosa existente. Igualmente, los métodos de clasificación más sencillos PCA-Projection y LDA resultan los más efectivos. De los métodos más sofisticados, el C-SVM resulta el más eficaz, aunque es sensible al tipo de semilla de iniciación utilizada. Se concluye que la espectroscopía NIRS combinada con técnicas quimiométricas es un método válido para clasificar corderos en matadero en función de su alimentación.

\section{A comparison of chemometrics classification tools for identification of perirenal fat in lambs}

\section{SUMMARY}

\section{ADDITIONAL KEYWORDS}

Lamb.

Perirenal fat.

NIRS.

Unsupervised/supervised classification.

INFORMATION

Cronología del artículo.

Recibido/Received: 03.07.2019

Aceptado/Accepted: 19.12.2018

On-line: 15.01 .2020

Correspondencia a los autores/Contact e-mail:

nutpedro@unex.es

\section{INTRODUCCIÓN}

La alimentación de los corderos durante el cebo puede hacerse de diferentes maneras en función del país de origen, disponibilidad de pastos, peso al sacrificio, precio de las materias primas para alimentación, etc (Alfonso et al. 2001). A priori, cabe suponer que todos estos factores, solos o combinados, pueden modificar las características de calidad de la carne de cordero en cuanto a sus atributos sensoriales y ello puede ser apreciado por el consumidor, manifestando algún tipo de preferencia (Font i Furnols et al. 2006). De ser así, ello conlleva un estudio químico complejo en la carne para tratar de evidenciar la causa de dichos cambios y preferencias (Demirel et al. 2006; Turner et al. 2014), además de mantener paneles de cata entrenados (Jaworska et al. 2016), todo ello cuesta tiempo y dinero.

La tecnología NIRS permite concentrar en un espectro la información física y química de la muestra analizada (McClure 2007), con el valor añadido 
de requerir una mínima preparación de la muestra, lo que ha hecho que sea una técnica ampliamente utilizada en la determinación de la calidad en industria de los alimentos (Cen \& He 2007), así como en distintas fases de la producción animal, desde los alimentos de los animales (Tejarina et al. 2018a), hasta los productos obtenidos de los mismos (Tejerina et al. 2018b, Croveti et al. 2018). En todo ello se va incorporando al análisis on line en muchos procesos (Lee 2007).

Por otra parte, la utilización de esta técnica, requiere el uso de herramientas quimiométricas que tratan de aplicar distintos métodos de regresión, como método alternativo al análisis químico convencional (Cen \& He 2007, Westad et al. 2013). De esta manera, a partir de un espectro, aplicando modelos de predicción desarrollados previamente, es posible estimar con una exactitud razonable la composición química de una muestra. Además, un solo espectro permite determinar simultáneamente tantos como ecuaciones de predicción tengamos.

Menos habitual ha sido la utilización de esta técnica para el análisis cualitativo de datos, de manera que sean las características espectrales de las muestras, las que permitan establecer la posible existencia de subgrupos con características propias y diferenciadas del resto. Para ello se requiere la aplicación de técnicas quimiométricas específicas que parten de sistemas de clasificación no supervisados que, en su caso, evolucionan a sistemas clasificados (Bevilacqua et al. 2013).

Hasta el momento, fundamentalmente se ha trabajado en análisis de carnes con un gran mercado y repercusión económica: vacuno, porcino y aves, centrándose en análisis de características químicas como humedad, grasa, proteína o perfil de ácidos grasos (Huang et al. 2008; Prieto et al. 2009). Y es en estas carnes donde se han desarrollado propuestas iniciales de clasificación (Bath et al. 2015, Prieto et al. 2009).

En carnes de menor demanda, en general, los trabajos de investigación con NIRS se centran en el ámbito de predecir su composición química. En carne de conejos (Pla et al. 2004, Riovanto et al. 2009, Zomeño et al. 2012), desarrollan ecuaciones de predicción específicas para humedad, proteína, grasa y ácidos grasos mayoritarios. En carne de corderos la situación es similar (Cozzolino et al. 2000, Guy et al. 2011), al igual que en carne caprina (Teixeira et al. 2015).

La utilización de espectroscopía NIR con técnicas quimiométricas de clasificación resulta especialmente útil en la industria, ya que abre la posibilidad de comparar muestras procedentes de diferentes tipos de producción con un patrón de referencia, permitiendo aplicar de facto un sistema de control de calidad automatizado, sin necesidad de recurrir a técnicas analíticas específicas, pero costosas.

El presente trabajo tiene como objetivo valorar la capacidad de la espectroscopía NIRS para detectar cambios en la composición de la grasa perirrenal de corderos de cebo, debidos a la alimentación recibida, y servir de base a un posible sistema de clasificación de canales en matadero basado en un patrón de referencia.

\section{MATERIAL Y MÉTODOS}

\section{CORDEROS Y SU ALIMENTACIÓN:}

Se parte de dos lotes de corderos de cebo procedentes de cebaderos comerciales de la cooperativa OVISO Soc. Coop. Ltda., cuya actividad se centra en la comarca de La Serena, Badajoz, S.O. España. Los corderos han sido de tipo merino, con un peso medio a la entrada en cebadero de $15 \mathrm{~kg}$ y un peso medio al sacrifico de $28 \mathrm{~kg}$, con unas estancia de 45 días en cebadero.

La diferencia entre ambos lotes ha sido el tipo de alimentación disponible durante el periodo de cebo. El lote testigo o de referencia se alimentó conforme a las normas de manejo habituales en estas explotaciones: paja de cereal a libre disposición y concentrado comercial. La paja de cereal tenía la siguiente composición sobre materia seca: $92.15 \%$ de materia seca, $6.66 \%$ de cenizas, $3.70 \%$ de proteína bruta, $1.96 \%$ de grasa bruta y $45.65 \%$ de fibra bruta. El concentrado tenía la siguiente composición sobre materia seca: $90.27 \%$ de materia seca, $6.67 \%$ de cenizas, $17.75 \%$ de proteína bruta, $2.65 \%$ de grasa bruta y $5 \%$ de fibra bruta.

El lote alternativo se alimentó mediante concentrado sin paja ("núcleo"), basado en el uso de grano entero de cebada (75\%) mezclado con el "núcleo" (25\%), que aporta proteína y corrector vitamínico mineral. La composición de la cebada fue la siguiente sobre materia seca: $90.11 \%$ de materia seca, $2.5 \%$ de cenizas, $12.15 \%$ de proteína bruta, $1.84 \%$ de grasa bruta y $5.33 \%$ de fibra bruta. La composición del "núcleo" concentrado fue la siguiente. La composición del núcleo fue la siguiente sobre materia seca: $90.13 \%$ de materia seca, $16.43 \%$ de cenizas, $37.39 \%$ de proteína bruta, 1.93\% de grasa bruta y $5.94 \%$ de fibra bruta.

\section{Muestras DE GRASA Y ESPECTRO NIRS:}

Sobre 26 corderos de cada uno de los lotes, tras 24 horas de oreo, se extrajo una muestra de grasa perirrenal. Las muestras se llevaron refrigeradas al laboratorio y fueron homogeneizadas mediante un molinillo de cuchillas.

Cada muestra se depositó en una caja de Petri de vidrio, que se rellenó con la ayuda de una espátula. De cada una de las muestras se obtuvo su espectro NIRS por duplicado, utilizando dos cajas de Petri, mediante un espectrofotómetro BUCHI-NirFlex 500. El espectro original se obtuvo en modo de reflectancia $R$, en el rango 1000-2500nm, con un total de 1501 puntos espectrales.

Transformaciones espectrales y análisis quimiométrico:

El análisis de los resultados se ha efectuado de manera paralela tanto con el formato espectral original (Reflectancia/R) como con la transformación logarítmica en Absorbancia ( $\mathrm{A}=1 / \mathrm{R})$.

Debido a la evidente presencia de efectos dispersivos en los espectros NIR (Figuras 1 y 2), se optó por una transformación EMSC (Afseth \& Kholer 2012) sobre cada una de las formas espectrales originales (R-EMSC y A-EMSC), para reducir su indefinición (Figura 3 y 4 ). 


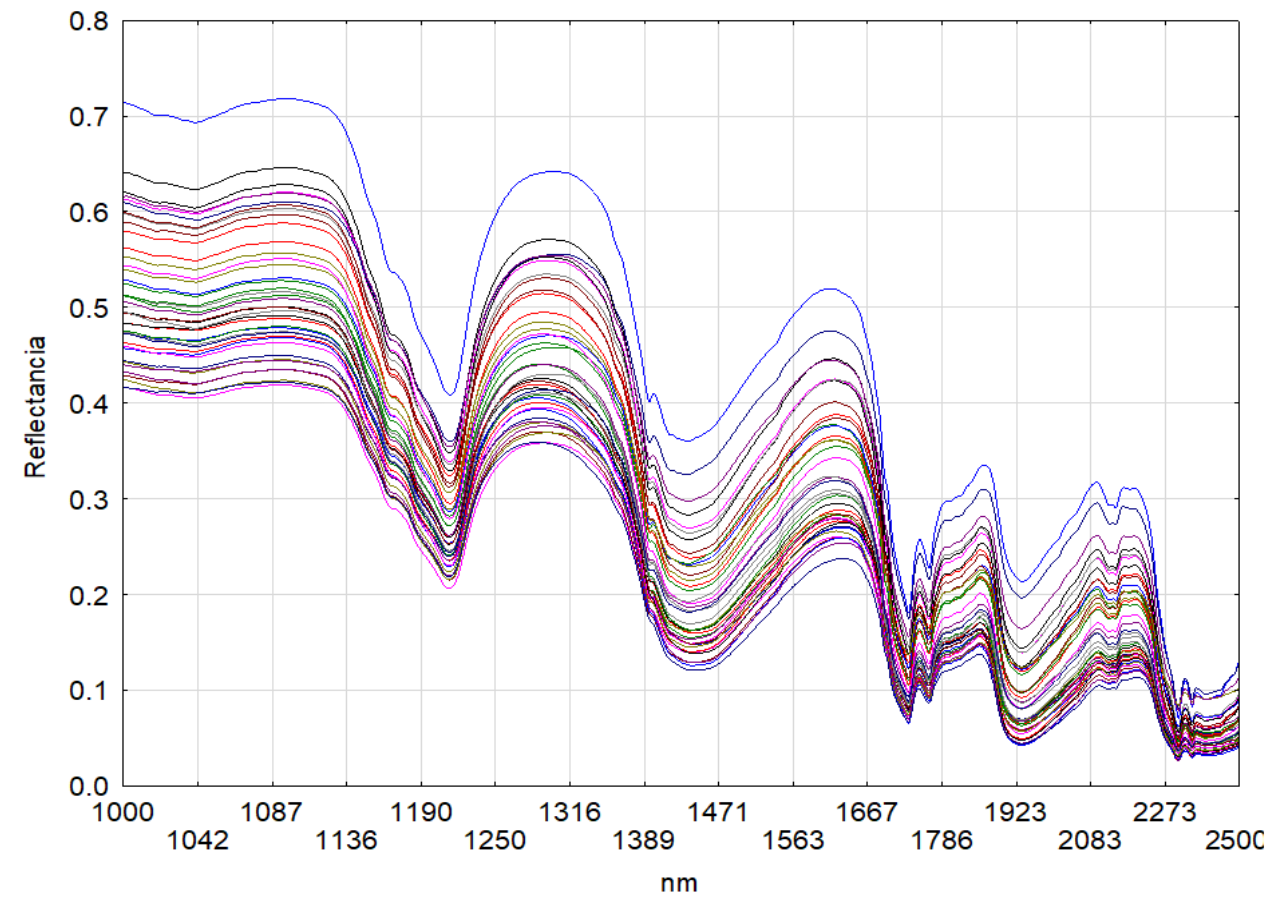

Figura 1. Espectros nirs originales en modo reflectancia $(1000-2500 \mathrm{~nm})$ (Original nirs spectra in reflectance mode (10002500nm).

Primeramente, se efectuó un análisis PCA sobre cada uno de los grupos de grasa, seleccionando en cada caso 20 muestras de calibración y 6 de validación mediante el algoritmo de Kennard-Stone, que recoge las muestras más representativas en el espacio de puntuación definido por los dos primeros componentes principales (Daszykowski et al. 2003). De esta manera se tienen 40 muestras de calibración y 12 de validación en total, la mitad perteneciente a cada grupo de corderos.
Como método inicial de clasificación se efectuó un análisis PCA de los espectros de calibración, y sobre la distribución de las muestras en el Score Plot, se procedió a proyectar las muestras de validación para comprobar su ubicación. De esta manera se construye un método elemental no supervisado de clasificación.

Posteriormente se procedió a elaborar distintos modelos de clasificación supervisada: SIMCA (Sof Independent Modelling Class Analogy), PLS-DA (Análisis Discriminante mediante Mínimos Cuadrados Parcia-

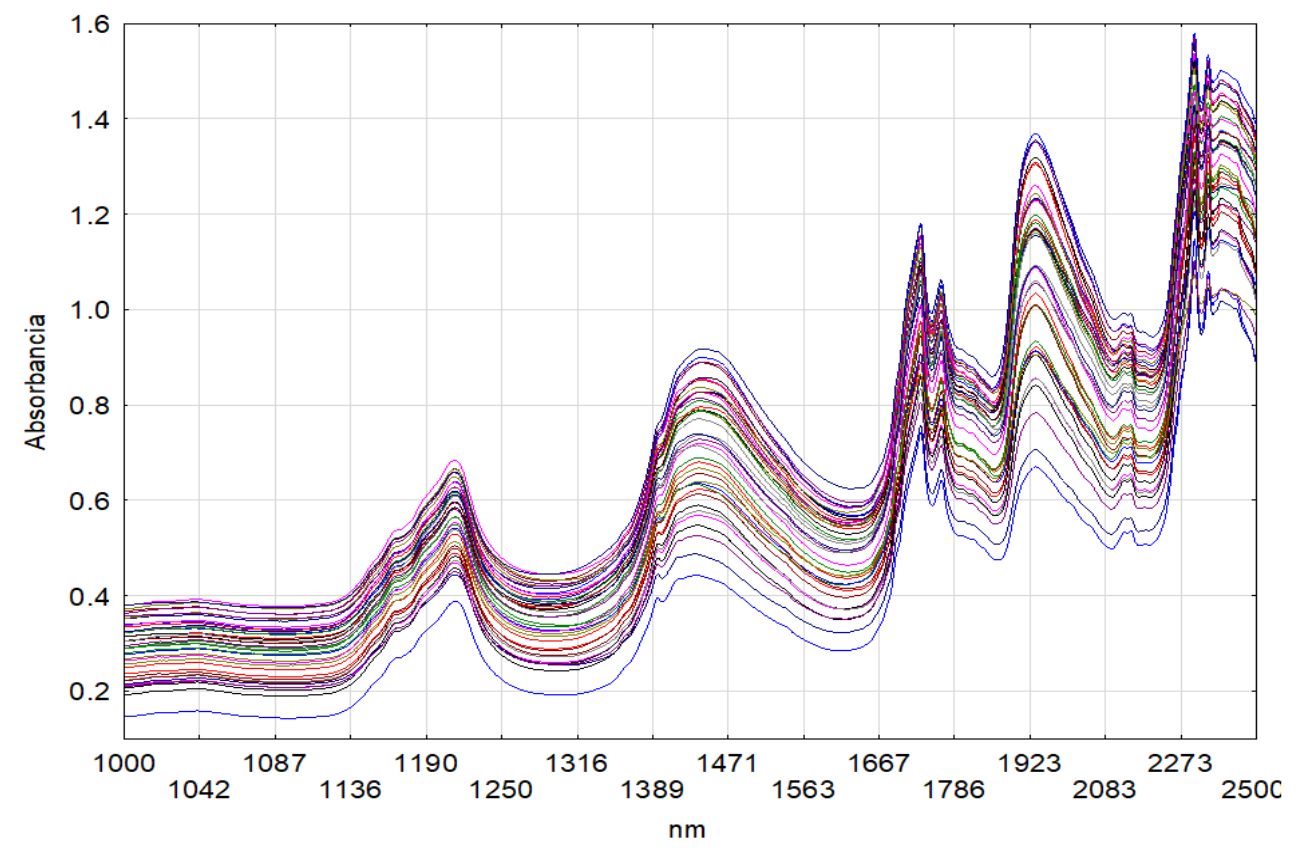

Figura 2. Espectros nirs originales en modo absorbancia (1000-2500nm) (Original nirs spectra in absorbance mode (10002500nm). 


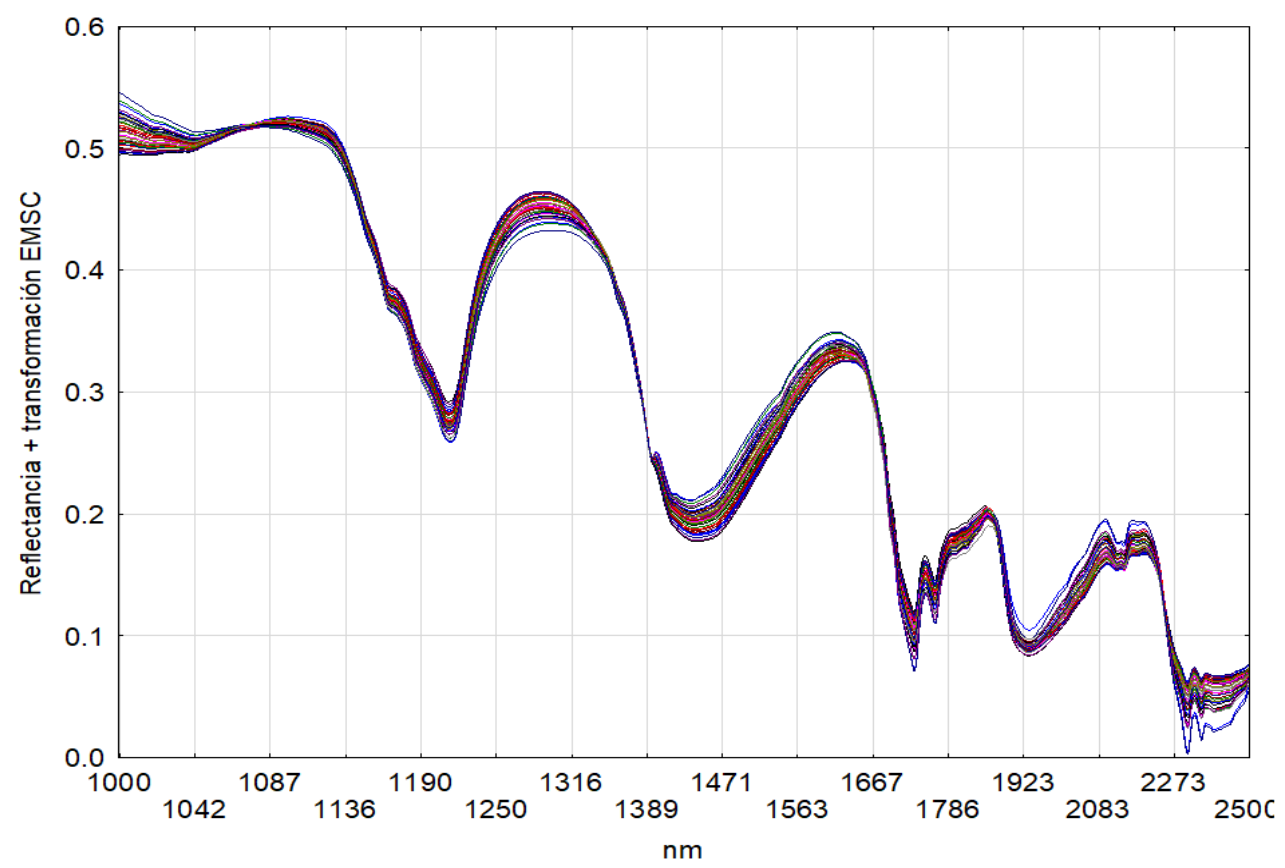

Figura 3. Espectros nirs originales en modo reflectancia tras transformación (1000-2500nm) (Original nirs spectra in reflectance mode after transformation (1000-2500nm).

les), LDA (Análisis Discriminante Lineal) y SVM-C (Clasificación mediante Máquinas de Soporte Vectorial).

SIMCA: trata de construir una regla de clasificación para " $\mathrm{n}$ " grupos conocidos, partiendo del espacio multidimensional que ocupa cada grupo, a partir de sendos análisis de componentes principales, funcionalmente maximiza el parecido entre individuos para definir las clases (Vanden \& Hubert, 2005).
PLS-DA: es un sistema de regresión multivariante (PLS, Matens \& Naes, 1993), en la que la variable a predecir es el grupo de pertenencia $(1=$ pertenece, $0=$ no pertenece) funcionalmente maximiza la diferencia entre individuos para definir las clases (Ballabio \& Todeschini, 2009).

LDA: utiliza una reducción previa de variables mediante PCA para, posteriormente, maximizar las diferencias entre categorías minimizando la varianza dentro de una categoría. Produce funciones discriminantes ortogonales entre sí que asignan cada muestra

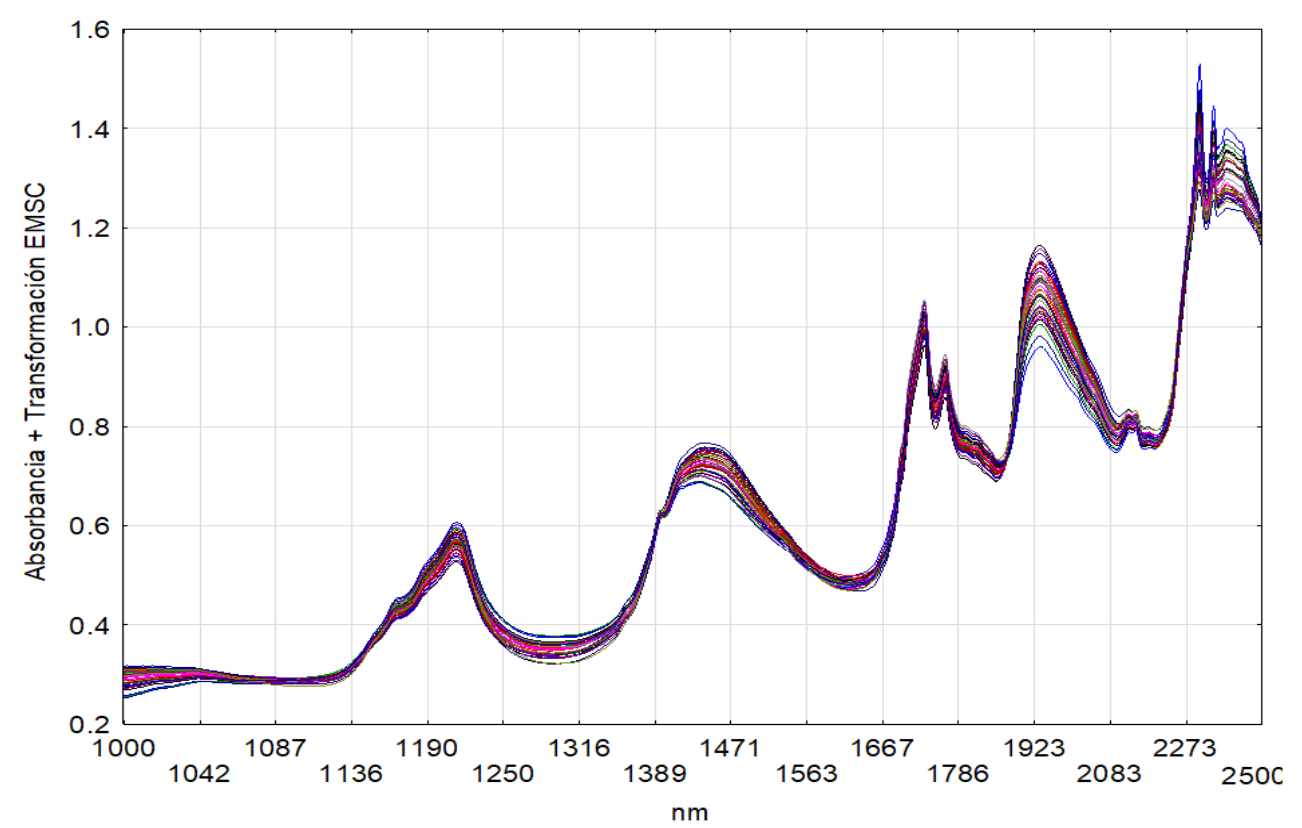

Figura 4. Espectros nirs originales en modo absorbancia tras transformación (1000-2500nm) (Original nirs spectra in absorbance mode after transformation $(1000-2500 \mathrm{~nm})$. 


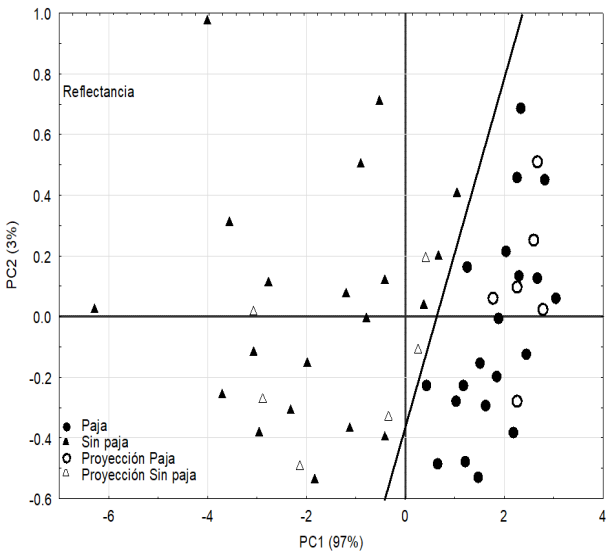

Figura 5. Gráfico de puntuación y proyección de muestras de validación en modo reflectancia (Graph of score and projection of validation samples in reflectance mode).

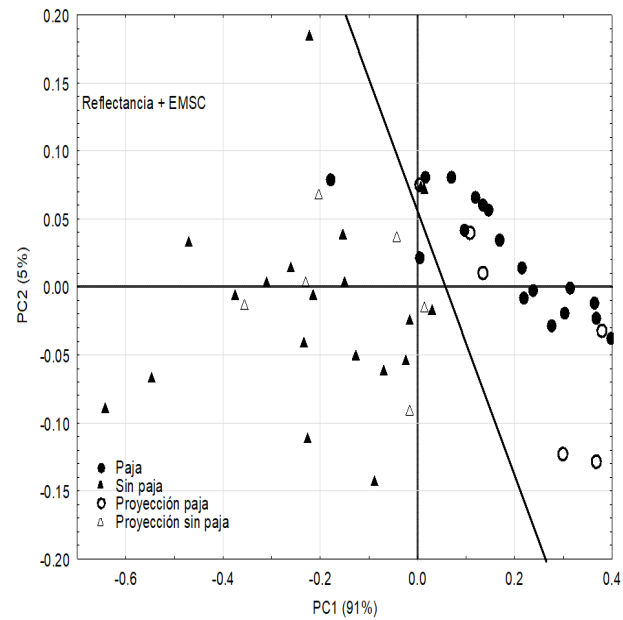

Figura 7. Gráfico de puntuación y proyección de muestras de validación en modo reflectancia + transformación EMSC (Graph of score and projection of validation samples in reflectance + EMSC transformation mode).

a uno de los grupos (Liu et al. 2006), pueden utilizarse 3 medidas de distancia entre grupos: lineal, cuadrática y Mahalanobis (Naes et al. 2002).

SVM-C: es un método de clasificación especialmente adaptado a separar dos clases entre ellas, se basa en funciones de clasificación lineal que construyen un hiperplano que maximiza la separación entre las clases propuestas (Ballabio \& Todeschini, 2009). La semilla para iniciar el cálculo puede ser lineal, polinómica, radial o sigmoidea (Hsu et al. 2010).

La habilidad de clasificación de los modelos se establece a través de los siguientes estadísticos asociados a las muestras de validación (Garrido et al. 2000):

- Error de Clasificación, porcentaje de muestras del conjunto de validación incorrectamente clasificadas.

- Sensibilidad, proporción de objetos que perteneciendo a la categoría son reconocidos correctamente por el modelo.

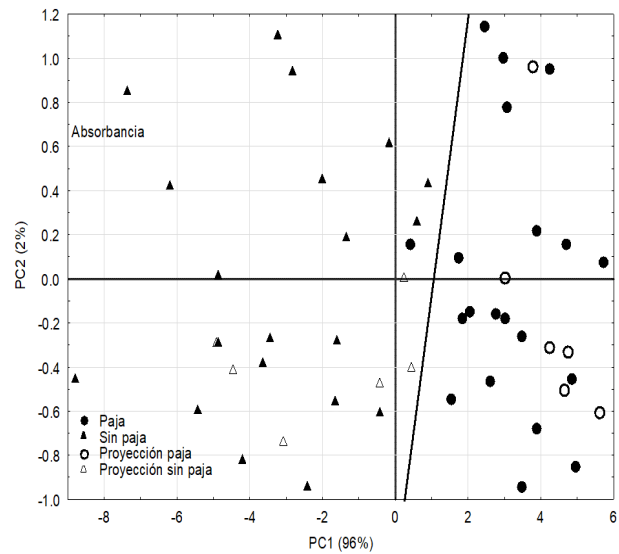

Figure 6. Gráfico de puntuación y proyección de muestras de validación en modo absorbancia (Graph of score and projection of validation samples in absorbance mode).

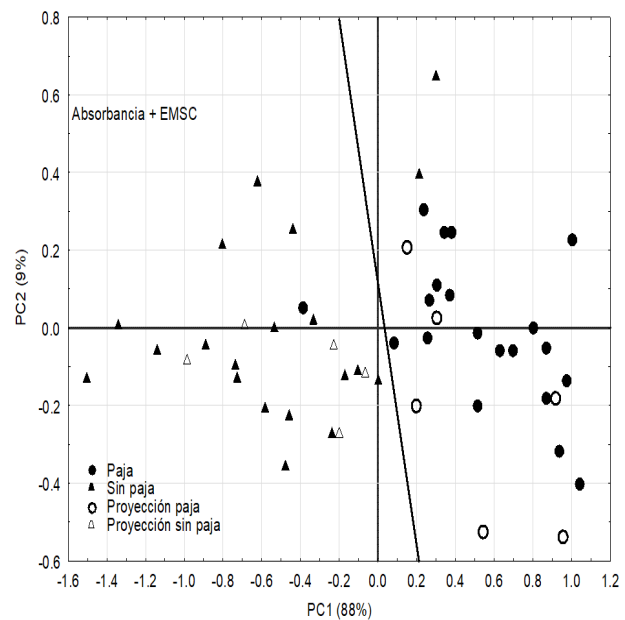

Figura 8. Gráfico de puntuación y proyección de muestras de validación en modo absorbancia + transformación EMSC (Graph of score and projection of validation samples in absorbance + EMSC transformation mode).

- Especificidad, proporción de objetos que no perteneciendo a la categoría así son reconocidos por el modelo.

Las distintas transformaciones espectrales, y los modelos quimiométricos se han desarrollado sobre el software The Unscrambler 10.5.

\section{RESULTADOS Y DISCUSIÓN}

Los modelos PCA separan adecuadamente ambos tipos de grasa utilizando sólo dos componentes principales en reflectancia, (Figura 5, símbolos negros), y tres en modo absorbancia (Figura 6, símbolos negros). La separación resulta peor en el caso de los espectros corregidos mediante EMSC, que tienen el óptimo en 3 componentes principales en ambos tipos de modo espectral (Figuras 7 y 8, símbolos negros). Ello sugiere que la textura física de la grasa genera efectos dispersivos en la luz infrarroja que, posiblemente, dificulten una calibración química convencional (Afseth \& Kholer 2012), pero a efectos de clasificación no se trata necesa- 
Tabla I. Índices estadísticos de los distintos métodos de clasifiación supervisada (Statistical indices of the different methods of supervised sclogging).

\begin{tabular}{lccccccccccccc}
\hline & \multicolumn{3}{c}{ Reflectancia } & \multicolumn{3}{c}{ Absorbancia } & \multicolumn{3}{c}{ R-EMSC } & \multicolumn{3}{c}{ A-EMSC } \\
\hline & E.C. & Sens. & Spec. & E.C. & Sens. & Spec. & E.C. & Sens. & Spec. & E.C. & Sens. & Spec. \\
\hline SIMCA & 58.33 & 91.67 & 41.67 & 41.67 & 83.33 & 58.33 & 16.67 & 83.33 & 83.33 & 16.67 & 91.67 & 83.33 \\
PLS-DA & 8.33 & 91.67 & 91.67 & 25.00 & 75.00 & 75.00 & 8.33 & 91.67 & 91.67 & 8.33 & 91.67 & 91.67 \\
LDA-Line. & 0.00 & 100.00 & 100.00 & 16.67 & 83.33 & 83.33 & 0.00 & 100.00 & 100.00 & 0.00 & 100.00 & 100.00 \\
LDA-Quad. & 0.00 & 100.00 & 100.00 & 0.00 & 100.00 & 100.00 & 8.33 & 91.67 & 91.67 & 0.00 & 100.00 & 100.00 \\
LDA-Maha. & 0.00 & 100.00 & 100.00 & 0.00 & 100.00 & 100.00 & 16.67 & 83.33 & 83.33 & 0.00 & 100.00 & 100.00 \\
SVM-Line. & 0.00 & 100.00 & 100.00 & 0.00 & 100.00 & 100.00 & 8.33 & 91.67 & 91.67 & 0.00 & 100.00 & 100.00 \\
SVM-Poly. & 41.67 & 58.33 & 58.33 & 8.33 & 91.67 & 91.67 & 25.00 & 75.00 & 75.00 & 25.00 & 75.00 & 75.00 \\
SVM-Radi. & 25.00 & 75.00 & 75.00 & 25.00 & 75.00 & 75.00 & 25.00 & 75.00 & 75.00 & 25.00 & 75.00 & 75.00 \\
SVM-Sigm. & 25.00 & 75.00 & 75.00 & 25.00 & 75.00 & 75.00 & 25.00 & 75.00 & 75.00 & 25.00 & 75.00 & 75.00 \\
\hline EC= Error de Clasificación, Sens. Sensibilidad, Spec. $=$ Specificidad & & & & & \\
\hline
\end{tabular}

riamente de imprecisiones ya que aporta información útil.

La proyección de las muestras de validación sobre el espacio definido por los dos primeros componentes principales, confirma estos resultados iniciales, de manera que son los espectros originales en modo reflectancia y absorbancia los que mejores resultados obtienen (Figuras 5 y $\mathbf{6}$, símbolos vacíos), mientras que en las proyecciones de los modelos EMSC la confusión es mayor (Figuras 7 y 8, símbolos vacíos).

El resultado de la aplicación de los distintos modelos supervisados se recoge en la Tabla I.

La clasificación SIMCA no resulta satisfactoria ya que, en el mejor de los casos, el error de clasificación es superior al $16 \%$. Con todo puede comprobarse que el tratamiento reductor de ruido (EMSC) resulta eficaz, incrementándose la selectividad de los modelos.

La clasificación mediante PLS-DA mejora, en todos los casos, el rendimiento de la clasificación SIMCA, pero no alcanza el $100 \%$ de efectividad.

La clasificación mediante LDA resulta satisfactoria en especial, ya que siempre hay algún procedimiento (Lineal/Quadratic/Mahalanobis) que produce el acierto pleno, al combinarse con alguna de las transformaciones espectrales. La clasificación resulta perfecta con los espectros originales en modo reflectancia, por lo que no serían necesarias más transformaciones, ahorrando trabajo y tiempo.

La clasificación SVM tiene una situación similar obteniéndose el mejor resultado con el procedimiento lineal y en modo reflectancia.

En conjunto, estos resultados sugieren que los métodos quimiométricos de clasificación sofisticados (SIMCA y PLS-DA) no resultan superiores al más tradicional LDA, lo que también es indicado por otros autores (Moscetti et al., 2015). El método SVM también resulta efectivo, pero es sensible a la semilla utilizada. En este sentido autores como Tan et al. (2009) o Balabin et al. (2009) también encuentran una mejor respuesta de SVM respecto a SIMCA o PLS-DA. Respecto al efecto del pretratamiento espectral para eliminar distorsiones, nuestros resultados no muestran una mejora significativa, al contrario que lo indicado por Kasemsumran et al. (2007), lo que sugiere que el pretramiento espectral es dependiente tanto del espectrofotómetro utilizado como del producto analizado, por lo que la optimización debe adaptarse a cada caso concreto (Pieszczek et al., 2018).

\section{CONCLUSIONES}

Mediante la espectroscopía NIR de grasa perirrenal es posible clasificar de manera correcta las canales de corderos en función de su alimentación, sin necesidad de un análisis químico específico. Esta técnica puede aplicarse en mataderos para completar la información comercial sobre las canales procesadas y comercializadas.

\section{AGRADECIMIENTOS}

Los autores agradecen al personal de OVISO su ayuda en la realización de este trabajo. Los costs de este trabajo han sido asumidos directamente por OVISO Soc. Coop. Ltda., Villanueva de la Serena, Badajoz, España.

\section{BIBLIOGRAFÍA}

Afseth, NK \& Kohler, A 2012, 'Extended multiplicative signal correction in vibrational spectroscopy, a tutorial', Chemometrics and Intelligent Laboratory Systems, vol. 117, pp 92-99.

Alfonso, M, Sañudo, C, Berge, P, Fisher, AV, Stamataris, C, Thorkelsson, G \& Piasentier, E. 2001, 'Influential factors in lamb meat quality. Acceptability of specific designations', In: Production systems and product quality in sheep and goats' Options Méditerranéennes: Série A. Séminaires Méditerranéens, vol 46, pp 19-28.

Balabin, RM, Safieva, RZ \& Lomakina, El 2011, Near-infrared (NIR) spectroscopy for motor oil classification: from discriminant analysis to supportvector machines', Microchemical Journal, vol. 98, pp. 121-128.

Ballabio, D \& Todeschini, R. 2009 'Multivariate classification for qualitative analysis', in D-W Sun (ed), Infrared Spectroscopy for food quality analysis and control. Academic Press, Burlington, USA. 
Bath, AA, Kumar, A, Para, PA, Kumar, S. \& Ganguly, S. 2015, 'Role of NIR Spectroscopy and Hyperspectral imaging in Meat Science: A Review', Int. J. Rec. Biotech., vol. 3, no. 3, pp. 30-39.

Bevilacqua, M, Bucci, R, Magrì, AD, Magrì, AL, Nescatelli, R \&Marini, F. 2013, 'Classification and class-modelling'. In F Marini (ed), Data Handling in Science and Technology, Vol. 28. Chemometrics in food chemistry. Elsevier, Oxford U.K.

Cen, H \& He, Y. 2007, 'Theory and application of near infrared reflectance spectroscopy in determination of food quality', Trends in Food Science \& Technology, vol. 18, pp 72-83.

Cozzolino, D, Murray, I, Scaife, JR \& Paterson, R. 2000, 'Study of dissected lamb muscles by visible and near infrared reflectance spectroscopy for composition assessment', Animal Science, vol. 70, no. 3, pp 417-423.

Daszykowski, M, Walczak, B \&Massart, DL. 2002, 'Representative subset selection', Analytica Chimica Acta, vol. 468, pp 91-103.

Demirel, G, Ozpinar, H, Nazli, B \& Keser, O. 2006, 'Fatty acids of lamb meat from two breeds fed different forage: concentrate ratio', Meat Sci., vol. 72, pp. 229-235

Font i Furnols, M, San Julián, R, Guerrero, L, Sañudo, C, Campo, MM, Olleta, JL, Oliver, MA, Cañeque, V, Álvarez, I, Díaz, MT, Brandscheid, W, Wicke, M, Nute, GR \& Montossi, F. 2006, 'Acceptability of lamb meat from different producing systems and ageing time to German, Spanish and British consumers', Meat Sci., vol. 72, pp. 545-554.

Garrido, A, García-Olmo, J \& De Pedro, E. 2000 'Espectroscopía de infrarrojo cercano (NIRS). Una metodología para implementar en sistemas de aseguramiento de la calidad y trazabilidad de productos derivados del cerdo ibérico', Sólo Cerdo Ibérico, vol. 4, pp. 39-44.

Crovetti, A.; Sirtori, F.; Aquilani, C.; Franci, O.; Bozzi, R. 2018. Predictive ability of FT-NIRS in the assessment of chemical composition of pork seasoned products. Arch. Zootec. PROCEEDINGS IX Simposio Internacional sobre el Cerdo Mediterráneo: 151-154.

Guy, F, Prache, S, Thomas, A., Bauchart, D. \& Andueza, D. 2011, 'Prediction of lamb meat fatty acid composition using near-infrared reflectance spectroscopy (NIRS)', Food Chem., vol. 127, pp 1280-1286.

Hsu, C.W., Chang, C.C. \& Lin, C.J. 2010, 'A practical guide to support vector classification' https://www.csie.ntu.edu.tw/ cilin/.

Huang, H, Yu, H, Xu, H \& Ying, Y 2008, Near infrared spectroscopy for on/in-line monitoring of quality in foods and beverages: a review', Journal of Feed Eng., vol.87, pp. 303-313.

Jaworska, D, Czauderna, M, Przybylsk,i W \& Rozbicka-Wieczorek, AJ 2016, 'Sensory quality and chemical composition of meat from lambs fed diets enriched with fish and rapeseed oils, carnosic acid and seleno-compounds', Meat Sci., vol. 119, pp. 185-192.

Kasemsumran, S, Thanapase, W \& Kiatsoonthon, A. 2007, 'Feasibility of Near-Infrared Spectroscopy to Detect and to Quantify Adulterants in Cow Milk', Analytical Sciences, vol. 23, pp. 907-910.

Lee, KA. 2007 'On-Line analysis in food engineering' In: Y Ozaki, WF McClure, Christy A.A. (ed) Near-Infrared Spectroscopy in food science and technology. John Wiley \& Sons, New Jersey, USA.

Liu, L, Cozzolino, D, Cynkar, WU, Gishen, M \& Colby, CB 2006, 'Geographic classification of Spanish and Australian Tempranillo red wines by visible and near-infrared spectroscopy combined with multivariate analysis', J. Agric. Food Chem., vol. 54, pp 6754-6759.

Martens, H \& Naes, T 1993, 'Multivariate calibration' John Wiley \& Sons, Chichester, U.K.

Naes, T; Isaksson, T; Fearn, T \& Davies, T 2002, 'A User-friendly guide to multivariate calibration and classification', NIR Publications, Chichester UK.
McClure, WF 2007, 'Introduction', In: Y Ozaki, WF McClure, Christy A.A. (ed). Near-Infrared Spectroscopy in food science and technology. John Wiley \& Sons, New Jersey, USA.

Moscetti, R, Haff, RP, Stella, E, Contini, M, Monarca, D, Cecchini, M, \& Massantini, R 2015, 'Feasibility of NIR spectroscopy to detect olive fruit infested by Bactrocera oleae', Postharvest Biology and Technology, vol. 99, pp. 58-62.

Pieszczek, L, Czarnik-Matusewicz, H \& Daszykowski, M 2018, 'Identification of ground meat species using near-infrared spectroscopy andclass modeling techniques-Aspects of optimization and validation using aone-class classification model', Meat Science, vol. 139, pp. 15-24.

Pla, M, Pascual, M \& Ariño, B 2004, 'Protein, fat and moisture content of retail cuts of rabbit meat evaluated with the NIRS methodology', World Rabbit Sci., vol. 12, pp. 149-158.

Prieto, N, Roehe, R, Lavín, P, Batten, G \& Andrés, S 2009, 'Application of near infrared reflectance spectroscopy to predict meat and meat products quality: A review', Meat Sci., vol. 83, pp. 175-186.

Riovanto, R, Szendrö, Z, Mirisola, M, Matics, Z, Berzaghi, P \& Dalle Zote, A 2009, 'Near Infrared Spectroscopy (NIRS) as a tool to predict meat chemical composition and fatty acid profile in different rabbit genotipes', Ital. J. Anim. Sci., vol. 8, no. 2, pp. 799-801.

Tan, C, Qin, X, \& Li, M 2009, 'Comparison of chemometric methods for brand classification of cigarettes by near-infrared spectroscopy. Vibrational Spectroscopy, vol. 51, pp. 276-282.

Teixeira, A, Oliveira, A, Paulos, K, Leite, A, Marcia, A, Amorim, A, Pereira, E, Silva, S \& Rodigues, S 2015, 'An approach to predict chemical composition of goat Longissimus thoracis et lumborum muscle by Near Infrared Reflectance spectroscopy', Small Rum. Res., vol. 126, pp. 40-43.

Tejerina, D.; García-Torres, S.; Martín-Tornero, E.; Gordillo, A.; Ortiz, A.; Ferraz-de-Oliveira, M.I., Machado, G.; Sales-Baptista, E.; Cabeza de Vaca, M. and Romero-Fernández, M.P. $2018^{a}$ NIRS potential use for the determination of natural resources quality from dehesa (acorn and grass) in Montanera system for Iberian pigs. Arch. Zootec. PROCEEDINGS IX Simposio Internacional sobre el Cerdo Mediterráneo: 71-73

Tejerina, D.; García-Torres, S.; Cabeza de Vaca, M.; Ortiz, A. and Romero-Fernández, M.P

$2018 \mathrm{~b}$. Evaluation of near-infrared spectroscopy (NIRS) for the quality control of packaged

cured ham-sliced from Iberian pigs. Arch. Zootec. PROCEEDINGS IX Simposio Internacional sobre el Cerdo Mediterráneo: 231-234

Tuner, KE, Belesky, DP, Cassida, KA \& Zerby, HN 2014, 'Carcass merit and meat quality in Suffolk lambs, Katahdin lambs, and meat-goat kids finished on a grass-legume pasture with and without supplementation', Meat Sci., vol. 98, pp. 211-219.

Vanden, K \& Hubert, M 2005, 'Robust classification in high dimensions based on the SIMCAMethod', Chemometrics and Intelligent Laboratory Systems, vol. 79, pp. 10-21.

Westad, F, Bevilacqua, M. \& Marini, F 2013, 'Regression' In: F Marini (ed). Data Handling in Science and Technology, Vol. 28. Chemometrics in food chemistry. Elsevier, Oxford U.K.

Zomeño, C, Juste, V \& Hernández, P 2012, 'Application of NIRS for predicting fatty acids in intramuscular fat of rabbit' Meat Science, vol. 91(2), pp. 155-159. 